\title{
How did you like 2017? Detection of language markers of depression and narcissism in personal narratives
}

\author{
Eva-Maria Rathner ${ }^{1}$, Julia Djamali ${ }^{1}$, Yannik Terhorst ${ }^{1}$, Björn Schuller ${ }^{2,3}$, Nicholas Cummins ${ }^{2}$, \\ Gudrun Salamon ${ }^{4}$, Christina Hunger-Schoppe ${ }^{5}$, Harald Baumeister ${ }^{1}$ \\ ${ }^{1}$ Clinical Psychology and Psychotherapy, University of Ulm, Germany \\ ${ }^{2}$ ZD.B Chair of Embedded Intelligence for Health Care and Wellbeing, University of Augsburg, Germany \\ ${ }^{3}$ GLAM - Group on Language, Audio \& Music, Imperial College London, UK \\ ${ }^{4}$ Sigmund Freud University, Vienna, Austria \\ ${ }^{5}$ Institute of Medical Psychology, University of Heidelberg, Germany \\ eva-maria.rathnereuni-ulm.de
}

\begin{abstract}
Language analyses reveals crucial information about an individual's current state of mind. Maladaptive psychological functioning appears in cognition, emotional experience and behaviour. In the time of the internet of things, a vast number of text and speech is available; subsequently, the interest in the automated detection of psychological functioning via language is rising. The current study indicates that depression and narcissism can be predicted through word use in personal narratives. Both conditions are characterised by an altered word count regarding anxiety and we (LIWC-based). While depressive individuals use less social words and more anxietyrelated words, narcissists do the opposite. This might reflect the verbal correlate of the cognitive triad in depression. In contrast, narcissists' word use mirrors their excommunicated anxiety of being an undesired self and their inability to reach long-term goals due to a lack of impulse control. The automated recognition of mental state through word use could improve early detection of mental disease, monitoring of disease course, delivery of tailored interventions and evaluation of therapy outcome.
\end{abstract}

Index Terms: free writing task, language use, narcissism, depression, LIWC

\section{Introduction}

The fundamental components of human language are linked with the particular nature of an individual's mind. The way we express ourselves reveals crucial information about our age, gender and state of mind [1]. Through language, we express social bonds, exchange knowledge and expand our point of view. The so-called cognitive revolution formed the basis for the development of psycholinguistic, neurolinguistic and interdisciplinary fields like neurosciences [2].

The upswing of computer sciences and technological interventions supports psychological research on the relation between mental processes and speech on a large scale. The use of online surveys allows researchers to collect data independently from the investigator's location and to cover a broader range of potential recruits [3]. Furthermore, social media grants access to an almost unlimited amount of personal data. People share their experiences, goals, wishes; reveal various interests, their current residence; and, even display political views [4]. Such data can be used to gain insight into the physical and mental health status of a person.

Differences in specific linguistic and paralinguistic patterns are found between individuals with mental health issues and healthy persons [5], [6]. Moreover, linguistic markers allow a prediction of mental disorders [7], [8]. These findings imply the potential use of automated language pattern detection for early diagnosis and the monitoring of high-risk populations [9].

Depression is characterized by lethargic, negative mood and automatized dysfunctional beliefs about oneself, the environment and the future [10]. This self-referencing ruminative negative state of mind of depressive individuals results in the frequent use of first person words [7], [11]. Depressive individuals tend to use negative emotion words more frequently when compared to healthy individuals [12]. Moreover, first person plural pronouns seem to be negatively associated with interpersonal distress and depressive symptoms [11]. These effects may result from a positive relationship between depression and self-focused attention [13]. However, specific language patterns, such as a higher use of first person singular pronouns in depressive individuals [7], are also associated with narcissism [14].

The narcissistic personality disorder is characterised by deficits in emotional empathy, pathological egocentrism, ideas of grandiosity, attention seeking [15] and impulsivity [16]. Moreover, narcissist individuals seem to experience increased hostility from comparison [17] and show elevated aggression in reaction to ego-threatening feedback [16]. Such a state of mind may manifest in linguistic patterns like an elevated use of first person singular pronouns [14], a more frequent use of sexual words [18], or a rare use of words related to affiliation and social connection [19]. Furthermore, narcissistic individuals use more antisocial, swear and anger words [19], [18], and less positive emotion words. Interestingly, there are no disparities between individuals with high versus low narcissism in the use of anxiety-related and sad words [19].

In summary, negative emotion words and first person pronouns as linguistic markers may represent a potential common factor for depressive and narcissistic individuals; pointing out the need of differential diagnostical word markers. However, these correlates do not refer to the sum of negative emotion words, but are rather restricted to single categories. In line with this, our research question for the differential 
Table 1: Demographic characteristics of the sample.

\begin{tabular}{ll}
\hline Variable & $M=22.6(S D=5.34)$ \\
\hline Age & \\
Gender & $57(25.9 \%)$ \\
Male & $162(73.6 \%)$ \\
Female & \\
Education level & $21(9.6 \%)$ \\
$\quad 10^{\text {th }}$ grade & $160(73.4 \%)$ \\
Undergraduates & $37(17.0 \%)$ \\
University Degree & \\
Employment status & $169(76.8 \%)$ \\
Student & $6(2.7 \%)$ \\
Trainee & $39(17.7 \%)$ \\
Employed & $3(1.4 \%)$ \\
Self-employed & $1(0.5 \%)$ \\
None & $2(0.9 \%)$ \\
Other & \\
Marital status & $89(40.5 \%)$ \\
Single & $114(51.8 \%)$ \\
In relationship/ married & $17(7.7 \%)$ \\
Other & \\
Mental Status & $200(90.9 \%)$ \\
No life-time diagnosis & $16(7.3 \%)$ \\
Major Depression & $4(1.8 \%)$ \\
Other &
\end{tabular}

detection of depressive and narcissistic individuals explored in this paper are as follows: Research question 1: Can depressive symptoms be predicted through word use in personal narratives and delineated from narcissism? and Research question 2: Can narcissistic traits be predicted through word use in personal narratives?

\section{Methods}

The study took place at the Department of Clinical Psychology and Psychotherapy, at the University of Ulm, and was approved by the local Ethics Committee. In total, 220 individuals were recruited via flyers, the local trial management system, social networks and disorder-specific online forums. Eligibility criteria were age of consent $(>/=18)$ and German as mother tongue to avoid effects on language use caused by language deficits and generational differences on the writing task. All sample demographics are displayed in Table 1.

\subsection{Procedure}

Blinded participants gave informed consent on study participation. The cover story was "A study on the relation between autobiographic memory and language". Participants reported their gender, age, mother tongue, relationship status, education level, diagnosed mental disorders and dyslexia. Individuals were invited to reflect their past year and to describe their expectations for the upcoming year. They were instructed to complete the writing task alone and in a silent environment for 10-20 minutes. After finishing the essay, participants completed the NPI-d and BDI-II-questionnaires. Finally, participants were debriefed and received reimbursement with either $10 €$ or course credits.

\subsection{Beck Depression Inventory (BDI-II)}

The Beck Depression Inventory II (BDI-II, [20]; German adaptation [21]) was used to assess the presence and severity of depressive symptoms for every participant, corresponding to
Table 2: BDI and NPI means and standard deviations of the sample.

\begin{tabular}{lllll}
\hline Questionnaire & Mean & SD & Min & Max \\
\hline BDI-II & 9.36 & 9.13 & 0 & 47 \\
NPI & 42.25 & 10.74 & 17 & 76 \\
\hline
\end{tabular}

Table 3: Descriptive characteristics of general word use

\begin{tabular}{lllll}
\hline Category & Mean & SD & Min & Max \\
\hline Word Count & 365.79 & 183.34 & 53 & 871 \\
I & 12.96 & 12.87 & 0 & 96 \\
We & 14.64 & 28.83 & 0 & 79 \\
Family & 10.29 & 23.27 & 0 & 97 \\
Negate & 2.80 & 3.28 & 0 & 14 \\
Affect & 10.56 & 15.83 & 0 & 97 \\
Negative Emotion & 4.79 & 13.04 & 0 & 99 \\
Anxiety & 0.63 & 0.86 & 0 & 5 \\
Numbers & 1.04 & 0.99 & 0 & 6 \\
Future & 1.87 & 2.58 & 0 & 11 \\
Eating & 8.41 & 21.44 & 0 & 96 \\
\hline
\end{tabular}

DSM-IV criteria. It contains 21 items, which have to be selfrated on a four-point scale, which ranges from 0 (symptoms not present) to 3 (severe symptoms). The items measure eight affective and thirteen somatic symptoms during the last two weeks. The total score (0-63) is deduced by summing the weighted statements of all items [20]. Storch, Roberti and Roth [22] reported good convergent validity and an internal consistency of .90 for the BDI-II total scale. Reliability of BDIII in this sample was excellent $\left(\omega_{\text {total }}=.93\right)$. Mean scores, minimum, maximum and standard deviation are shown pictured in Table 2.

\subsection{Narcissistic Personality Inventory (NPI)}

The Narcissistic Personality Inventory (NPI-d [23]), based on Raskin and Terry's scale [24], measures narcissistic personality traits on a 17 item self-report scale on a five-point Likert scale from 0 ("not at all like me") to 4 ("totally like me"). Assuming one-dimensionality, a sum score can be calculated (scores 0 85 ). The German version of the NPI represents a valid and economical instrument with an internal consistency of .82 and a reliability of .69 to .88 [23]. The NPI-d [23] measures narcissistic personality, which is not to be confused with the narcissistic personality disorder (NPD [15]). Narcissistic personality tendencies represent a dimensional construct [25] and, in contrast to the NPD, do not necessarily require diagnosis or treatment. Reliability of NPI in the current sample was good $\left(\omega_{\text {total }}=.88\right)$. Values of the sample are presented in Table 2 .

\subsection{Linguistic Inquiry Word Count (LIWC)}

To analyse linguistic markers and dimensions of depression and narcissism, all essays were analysed using Linguistic Inquiry and Word Count, more specifically the German dictionary [26], [27]. LIWC has been used extensively for psychological text analysis. It calculates the percentage of each word category within the entire text. The basis for the classification is an integrated dictionary, which divides the words into categories and subcategories. The categories of the dictionary include linguistic and grammatical dimensions (e.g. pronouns, prepositions) as well as psychological processes like emotions, cognition and social categories [28].

A study on the psychometric qualities of the German 
adaption of the LIWC [27] found that the majority of LIWC categories were equivalent to the English original. Furthermore, it was shown that the German adaptation of LIWC is robust against writing errors [27], which is particularly important since the participants were instructed to write freely and unrestrained. Descriptive characteristics of the general word use are summarized in Table 3.

\subsection{Statistical analyses}

The reliability of BDI-II and NPI was assessed within the study to ensure a high quality assessment. Cronbach's alpha is the most popular measure for reliability, but has numerous deficiencies [29]. Thus, $\omega$ total was calculated [30] instead of alpha. Maximum Likelihood was used as estimator. Reliability for the BDI-II was excellent and good for the NPI.

To identify word categories predicting depressive symptoms (BDI-II) or narcissism (NPI), linear regression analysis was applied. In a first step, the word categories negative emotion, positive emotion, self-referencing words, physical state, grammatical categories and social words as well as their subcategories were included as predictors for BDI-II and NPI, respectively. In a stepwise process, all non-significant predictors were excluded. In each step, the predictor with the least incremental effect was removed. For all regression analyses, the ordinary least square estimator was used. Analyses were carried out using the software R [31] and the R package psych.

\section{Results}

\subsection{Prediction of depressive symptoms through word use}

The initial linear regression model when predicting depressive symptoms from word use contained 34 predictors $(\mathrm{F}(34$, $185)=1.61, p=.025$, adj. $\left.R^{2}=.087\right)$. Five predictors remained in the final model $\left(\mathrm{F}(5,214)=6.09, \mathrm{p}<.001\right.$, adj. $\left.\mathrm{R}^{2}=0.104\right)$ Estimates are summarized in Table 5. The model explains $10 \%$ of the variance.

The analysis indicates that depression score is associated with a higher use of the categories: I, we, negate, anxiety and eating. The domains Negate and Anxiety have the highest impact on BDI-II scores. Each increase of $1 \%$ of negation use in the personal narrative is associated with an increase of .18 standard deviations in the BDI-II. Analogously, each increase of $1 \%$ of anxiety-related words in the narrative is accompanied in the BDI-II by the factor of .19 standard deviations.

The higher use of negation in relation to a higher state of depressive symptoms is in line with the Beck's theory of the cognitive triad of depression [10], which describes an irrationally biased belief system of automatic, spontaneous and seemingly uncontrollable negative thoughts about oneself, the environment and the future. This negative belief system leads to a higher use of negations, mirroring the person's negatively blurred depressive state of mind, and results in a confirmation of that belief system over the alteration of perception, behaviour and emotion. Depression also alters brain structure [32], function and connectivity and vice versa [33].

Of note, earlier findings of a higher use of overall negative emotion words could not be replicated in the current study [34], [35]. A possible explanation is the diverse assessment of depression. We assessed depressive symptoms at an excellent reliability and used depression as a continuous variable in our models in a more naturalistic approach. Other studies used categorization (depressed vs. formerly depressed vs. healthy)
Table 5: Linear regression model to predict BDI-

II scores through language use (Model 1).

\begin{tabular}{lll}
\hline & Estimate & $\boldsymbol{P}$ \\
\hline Intercept & -.042 & $<.001$ \\
I & 0.01 & .018 \\
We & -0.03 & $<.001$ \\
Negate & $\mathbf{. 1 8}$ & $<.001$ \\
Anxiety & $\mathbf{. 1 9}$ & $\mathbf{. 0 3 2}$ \\
Eating & 0.01 & .024 \\
\hline $\mathrm{F}(5,214)=6.09, \mathrm{p}<.001, \mathrm{R}^{2}=0.125$, adj. $\mathrm{R}^{2}=0.104$
\end{tabular}

Table 6: Linear regression model to predict NPI scores through language use (Model 2).

\begin{tabular}{lll}
\hline & Estimate & $\boldsymbol{P}$ \\
\hline Intercept & .44 & $<.001$ \\
We & 0.04 & $<.001$ \\
Numbers & $\mathbf{- . 2 3}$ & $<.001$ \\
Affect & 0.01 & .017 \\
Negative emotion & .01 & .008 \\
Anxiety & $\mathbf{- . 3 6}$ & $<.001$ \\
Family & -0.02 & $<.001$ \\
Future & $\mathbf{- . 1 1}$ & $\mathbf{. 0 4 1}$ \\
Eating & -0.01 & .0139 \\
\hline $\mathrm{F}(8,211), \mathrm{p}<.001, \mathrm{R}^{2}=.177$, adj. $\mathrm{R}^{2}=.146$
\end{tabular}

with self-reported measurements or categorization via semistructured interviews (SCID-I \& SCID II) with complete case analysis. Both strategies imply methodological issues. Moreover, state-of-the-art treatment involves steady monitoring of depressive individuals, which could be realised with continuous measurements of severity-related language patterns. Such automatized language detection related to depression severity could be used as an early warning system to prevent relapse [36]. Relapse is a major driver of health related costs and personal burden in relation to depression. The study indicates that there might be changes in language use in relation to symptom severity resulting in the higher use of first-person pronouns in severely depressed individuals given the results on inpatients word use in Zimmerman's study [35] compared to our analysis with depression severity as a continuous variable.

Next to negations, anxiety related words contribute to the detection of depression state. Recently, the differential diagnostic approach in mental health has been questioned [37], and transdiagnostical approaches in treatment are on the rise. Especially as anxiety and depression share substantial neurological substrates. In line with this, a higher use of anxiety related words reflects the neurologic correlate of depressive symptoms.

Of note, we did not find a higher use of negative emotion words in association to depression scores as reported in the literature [7], [11]. Interestingly the effect of the use of $I$ was rather small although extensively reported in the literature [35] This attenuated use of $I$ is seen as a correlate of depressives' self-focused attention. Differences between our results and earlier studies on depressive language use might result from our rather non-depressive sample [35].

The findings therefore contribute to a language-based approach of early diagnosis and monitoring of high risk populations, which can complement healthcare systems. Hence, the embedding of automatized analysis of language use in webor mobile-based clinical treatments could improve therapy monitoring and outcome. 


\subsection{Prediction of narcissism through word use}

The initial linear regression model when predicting narcissism from word use model contained 34 predictors $(\mathrm{F}(34$ $185)=2.375, \mathrm{p}<.001$, adj. $\left.\mathrm{R}^{2}=.176\right)$. The final model was reduced to 8 predictors $\left(\mathrm{F}(8,211), \mathrm{p}<.001\right.$, adj. $\left.\mathrm{R}^{2}=.146\right)$ Estimates are summarized in Table 6. The model explains $14.6 \%$ of variance.

Results suggest that the level of narcissism is related to a more frequent use of the LIWC categories we, affect and negative emotion; and a lower use of the categories numbers, anxiety, family, future and eating. The influence of those LIWC categories on narcissism scores is slightly higher than the influence on word use on the BDI-II. For example, each increase of $1 \%$ of use of numbers in the personal narrative is associated with a decrease of 23 standard deviations in the NPI Analogous, each increase of $1 \%$ of anxiety-related words in the narrative is accompanied by a decrease in the NPI of .36 standard deviations and for future words .11 standard deviations. This result is highly interesting as it indicates that narcissistic language use can rather be characterised by what is not expressed (numbers, anxiety and future), than by which words are used.

Narcissism is strongly associated with a thrive for selfenhancement, aggressive behaviour and negative long-term outcomes [38]. Narcissism should be seen as a continuum from functional to dysfunctional forms [39]. In dysfunctional narcissist personality structures, a combination of high extraversion and low agreeableness leads to a number of interpersonal conflicts, resulting in narcissists aggressive selfdefeating behaviour [40]. There is a strong positive relationship between narcissism and impulsivity [38], indicating that the achievement of status as a long-term goal is hindered by a lack of self-control. This dynamic results in a lower use of futurerelated words; thinking about long-term goals reduces the narcissist's self-esteem. The reduction in self-esteem is caused by the individual's insight into their own inability to reach longterm goals. Nevertheless, they search for the fault in their environment and not themselves, leading to an inability to change.

A majority of this dynamic might be caused by the fear of being an undesired self [41]. This fear seems not bearable and not expressible. As a result, narcissism is correlated to a reduction in anxiety related word use. Additionally, we observed a higher use of emotion, specifically negative emotion words. Narcissistic individuals experience negative emotions more frequently due to their interpersonal problems [16]. This finding was only described as a trend in earlier studies, which assessed narcissism with a single item [19]. Furthermore, categorisation of low versus high narcissism was based on standard deviations in the given sample. These differences in assessment imply that our findings are more robust in regard to reliability.

The present study confirms earlier findings regarding a lower frequent use of affiliation and social connection words [19], which seems to express the narcissistic individuals' way of coping with an unbearable high thrive for social recognition. Of note, we could not confirm elevated first person use [14] Carey and colleagues [42] also questioned the elevated use of personal pronouns in a large-scale multi-centre study that tested five relevant communication contexts. We did not observe a higher use of sexual, antisocial, swear, sad and anger words [18]. We hypothesize that the use of those word categories is a reflection of narcissists' interpersonal problems. Those differences in word use associated with narcissism were reported for social network use [19]. Our free writing task did not involve any interaction with others, therefore, we might have not covered this facet of narcissistic word use in the current study.

\subsection{Limitations and further research}

The explorative approach of this study needs to be critically reflected. The exclusion of a predictor may for example, affect the significant influence of previously excluded predictors. However, it is not feasible to analyse every iteration, as in terms of our 34 predictors, 17,179,869,184 combinations are possible. Therefore, important outcomes have been depicted, but validation on a new sample has to be made. On the other hand, this approach minimises the risk to include irrelevant predictors.

Furthermore, age variance has been restricted intendedly to minimize confounding by age related differences in language use. However, through this control method, as well as the homogeneity regarding gender (see Table 1), the general applicability of the findings is limited. Only $25 \%$ of participants were male, therefore statistical analyses of gender effects were not applicable.

Further studies should use the current data set to study differential diagnosis between narcissism and depression with deep machine learning techniques.

\section{Conclusions}

The study contributes to the understanding of linguistic patterns in psychopathology. We conclude that both, depressive symptoms and narcissism level can be predicted through word use in personal narratives. Moreover, the findings indicate that the LIWC categories anxiety and we represent a potential distinctive factor for detection of depressive and narcissistic state. Furthermore, depression severity was associated with a higher use of negation, reflecting the cognitive triad of depression. Contrastingly, the level of narcissism was correlated with an extenuated use of numbers, anxiety-related words and the use of future; mirroring narcissists excommunicated anxiety of being an undesired self and inability to reach long-term goals due to a lack of impulse control. Further research leading to a profound knowledge about the language patterns of individuals' state on the depressive and narcissistic spectrum may facilitate early diagnosis, standardized monitoring of disease course, distribution of tailored interventions and evaluation of therapy outcome.

\section{Acknowledgements}

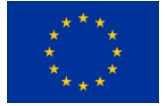

This research has received funding from the Innovative Medicines Initiative 2 Joint Undertaking under grant agreement No 115902. This Joint Undertaking receives support from the European Union's Horizon 2020 research and innovation programme and EFPIA.

The authors thank B. Fleischmann, C. Heiser, F. Nuber, J.Reinhardt, C. Sotiriadou and S. Spormann.

\section{References}

[1] J. W. Pennebaker, M. R. Mehl, and K. G. Niederhoffer, "Psychological aspects of natural language use: Our words, our selves,” Аnnu. Rev. Psychol., vol. 54, pp. 547-577, 2003.

[2] G. A. Miller, "The cognitive revolution: a historical 
perspective," Trends Cogn. Sci., vol. 7, no. 3, pp. 141-144, 2003.

[3] M. H. Birnbaum, "Human research and data collection via the internet," Annu. Rev. Psychol., vol. 55, no. 1, pp. 803-832, 2004.

[4] R. Gross and A. Acquisti, "Information revelation and privacy in online social networks," in Proceedings of the 2005 ACM workshop on Privacy in the electronic society, 2005, pp. 71-80.

[5] N. Cummins, S. Scherer, J. Krajewski, S. Schnieder, J. Epps, and T. F. Quatieri, "A review of depression and suicide risk assessment using speech analysis," Speech Commun., vol. 71, pp. $10-49,2015$

[6] F. Ringeval et al., "Real-life depression, and affect recognition workshop and challenge," in Proceedings of the 7th International Workshop on Audio/Visual Emotion Challenge 2017, 2017, pp. 3-9.

[7] M. De Choudhury, M. Gamon, S. Counts, and E. Horvitz, "Predicting Depression via Social Media," in Proceedings of the 7th International AAAI Conference on Weblogs and Social Media, 2013, vol. 2, pp. 128-138.

[8] B. Stasak, J. Epps, and R. Goecke, "Elicitation design for acoustic depression classification: An investigation of articulation effort, linguistic complexity, and word affect," in Proceedings INTERSPEECH 2017, 2017, pp. 834-838.

[9] J. P. Pestian et al., "A machine learning approach to identifying the thought markers of suicidal subjects: A prospective multicenter trial," Suicide life-threatening Behav., vol. 47, no. 1, pp. 112-121, 2017.

[10] A. T. Beck, J. A. Rush, B. F. Shaw, and G. Emery, Cognitive Therapy of Depression. NY, USA: Guilford Press, 1987.

[11] J. Zimmermann, M. Wolf, A. Bock, D. Peham, and C. Benecke, "The way we refer to ourselves reflects how we relate to others: Associations between first-person pronoun use and interpersonal problems," J. Res. Pers., vol. 47, no. 3, pp. 218-225, 2013.

[12] N. Ramirez-Esparza, C. Chung, E. Kacewicz, and J Pennebaker, "The psychology of word use in depression forums in English and in Spanish: Testing two text analytic approaches," in ICWSM, 2008, pp. 102-108.

[13] M. Nilly and J. Winquist, "Self-focused attention and negative affect: A meta-analysis," Psychol. Bull., vol. 128 , no. 4, pp. 638-662, 2002.

[14] R. Raskin and R. Shaw, "Narcissism and the use of personal pronouns," J. Pers., vol. 56, pp. 393-404, 1988.

[15] American Psychiatric Association, Diagnostic and statistical manual of mental disorders (DSM-5®). Washington, D.C: American Psychiatric Pub, 2013.

[16] T. S. Stucke and S. L. Sporer, "When a grandiose self-image is threatened: Narcissism and self-concept clarity as predictors of negative emotions and aggression following ego-threat," J. Pers., vol. 70, no. 4, pp. 509-532, 2002.

[17] L. M. Bogart, E. G. Benotsch, and J. D. P. Pavlovic, "Feeling superior but threatened: The relation of narcissism to social comparison," Basic Appl. Soc. Psych., vol. 26, no. 1, pp. 3544, 2004 .

[18] N. S. Holtzman, S. Vazire, and M. R. Mehl, "Sounds like a narcissist: Behavioral manifestations of narcissism in everyday life," J. Res. Pers., vol. 44, pp. 478-484, 2010.

[19] J. Goldbeck, "Negativity and anti-social attention seeking among narcissists on Twitter: A linguistic analysis.," First Monday, 2016.

[20] A. T. Beck, R. A. Steer, and G. K. Brown, BDI-II: Beck depression inventory. Pearson, 1996.

[21] M. Hautzinger, F. Keller, and C. Kühner, Beck depressionsinventar (BDI-II). Frankfurt, Germany: Harcourt Test Services, 2006

[22] E. A. Storch, J. W. Roberti, and D. A. Roth, "Factor structure, concurrent validity, and internal consistency of the Beck Depression Inventory - Second Edition in a sample of college students," Depress. Anxiety, vol. 19, no. 3, pp. 187-189, 2004.

[23] G. von Collani, "Modifizierte deutsche Versionen des
Narcissistic Personality Inventory (NPI-d)," in Zusammenstellung sozialwissenschaftlicher Items und Skalen. ZIS, Vol. 12., A. Glöckner-Rist, Ed. 2008.

[24] R. Raskin and H. Terry, "A principal-components analysis of the Narcissistic Personality Inventory and further evidence of its construct validity.," J. Pers. Soc. Psychol., vol. 54, no. 5, pp. 890-902, 1988.

[25] W. K. Campbell and J. D. Foster, "The narcissistic self: Background, an extended agency model, and ongoing controversies.," in The self, C. Sedikides and J. Spencer, Eds. London, GB: Psychology Press, 2007, pp. 115-138.

[26] J. W. Pennebaker and A Graybeal, "Patterns of natural language use: Disclosure, personality, and social integration," Curr. Dir. Psychol. Sci., vol. 10, no. 3, pp. 9093, 2001.

[27] M. Wolf, A. B. Horn, M. R. Mehl, S. Haug, J. W. Pennebaker, and H. Kordy, "Computergestützte quantitative Textanalyse: Äquivalenz und Robustheit der deutschen Version des Linguistic Inquiry and Word Count," Diagnostica, vol. 54 no. 2, pp. 85-98, 2008.

[28] Y. R. Tausczik and J. W. Pennebaker, "The psychological meaning of words: LIWC and computerized text analysis methods," J. Lang. Soc. Psychol., vol. 29, no. 1, pp. 24-54, 2010.

[29] T. J. Dunn, T. Baguley, and V. Brunsden, "From alpha to omega: A practical solution to the pervasive problem of internal consistency estimation," Br. J. Psychol., vol. 105, no. 3, pp. 399-412, 2014

[30] W. R. Revelle and R. Zinbarg, "Coefficients Alpha, Beta, Omega and GLB: Comments on Sijtsma," Psychometrika vol. 74, no. 1, pp. 145-154, 2009.

[31] R Core Team, "R: A language and environment for statistical computing.” R Foundation for Statistical Computing, Vienna, Austria, 2017.

[32] S. G. Costafreda, C. Chu, J. Ashburner, and C. H. Y. Fu, "Prognostic and diagnostic potential of the structural neuroanatomy of depression," PLoS One, vol. 4, no. 7, pp. 15, 2009.

[33] R. J. Zatorre, R. . Fields, and H. Johansen-Berg, "Plasticity in Gray and White: Neuroimaging changes in brain structure during learning," Nat. Neurosci., vol. 15, no. 4, pp. 528-536, 2013.

[34] S. S. Rude, E. M. Gortner, and J. W. Pennebaker, "Language use of depressed and depression-vulnerable college students," Cogn. Emot., vol. 18, no. 8, pp. 1121-1133, 2004 J. Zimmermann, T. Brockmeyer, M. Hunn, H. Schauenburg, and M. Wolf, "First-person pronoun use in spoken language as a predictor of future depressive symptoms: Preliminary evidence from a clinical sample of depressed patients," Clin Psychol. Psychother., vol. 24, no. 2, pp. 384-391, 2017.

[36] F. Mugica, A. Nebot, S. Bagherpour, L. Badalon, and A. Serrano-Blanco, "A model for continuous monitoring of patients with major depression in short and long term periods Francisco," Technol. Heal. Care, pp. 1-25, 2016.

[37] B. J. Casey, M. E. Oliveri, and T. Insel, "A neurodevelopmental perspective on the research domain criteria (RDoC) framework," Biol. Psychiatry, vol. 76, no. 5, pp. 350-353, 2014.

[38] S. Vazire and D. C. Funder, "Impulsivity and the self defeating behavior of narcissists," Personal. Soc. Psychol. Rev., vol. 10, no. 2, pp. 154-165, 2006.

[39] D. L. Paulhus, "Normal narcissism: Two minimalist accounts," Psychol. Inq., vol. 12, no. 4, pp. 228-230, 2001.

[40] G. Miller, E. Chen, and S. W. Cole, "Health psychology: developing biologically plausible models linking the socia world and physical health.," Annu. Rev. Psychol., vol. 60, pp. 501-524, 2009.

[41] M. D. Barnett and P. M. Womack, "Fearing, not loving, the reflection: Narcissism, self-esteem, and self-discrepancy theory," Pers. Individ. Dif., vol. 74, pp. 280-284, 2015.

[42] A. L. Carey et al., "Narcissism and the use of personal pronouns revisited," J. Pers. Soc. Psychol., vol. 109, no. 3, pp. e1-e15, 2015. 